\title{
Endophthalmitis Following Cataract Surgery: Clinical Features, Treatment and Prophylaxis
}

\author{
Avinash Pathengay, Stephen G. Schwartz, \\ Harry W. Flynn Jr. and Darlene Miller \\ Department of Ophthalmology, Bascom Palmer Eye Institute, University of Miami, \\ Miller School of Medicine, Miami, Florida
}

USA

\section{Introduction}

Acute-onset postoperative endophthalmitis is characterized by marked inflammation of intraocular fluids and tissues. Despite appropriate therapeutic intervention, endophthalmitis may result in severe visual loss. Postoperative endophthalmitis has been reported following every type of ocular surgery but because cataract surgery is the most commonly performed type of ocular surgery, this setting is the most frequent cause in clinical practice.

\section{Incidence}

The incidence of acute-onset postoperative endophthalmitis (Table 1) after cataract surgery has steadily declined from $2 \%$ in the first half of the twentieth century to current levels ranging from $0.03 \%$ to $0.21 \%$ (Leopold, 1971; Allen \& Mangiaracine, 1964; Aaberg et al., 1998; West et al., 2005; Jensen et al., 2008; Moshirfar et al., 2007; Freeman et al., 2010; Ravindran et al., 2009; Wykoff et al., 2010). The reported rate of delayed-onset (chronic) postoperative endophthalmitis following cataract surgery in one single-center series was $0.017 \%$ (Mezaine et al., 2009).

\begin{tabular}{|c|l|c|c|c|}
\hline & \multicolumn{1}{|c|}{ Author } & Years & No. of cases & Incidence $\%$ \\
\hline 1 & Leopold & $(1920-1940)$ & Meta-analysis & 2.00 \\
\hline 2 & $\begin{array}{l}\text { Allen \& } \\
\text { Mangiaracine }\end{array}$ & $(1958-1962)$ & $22 / 20,000$ & 0.09 \\
\hline 3 & Aaberg et al. & $(1984-1994)$ & $34 / 41,654$ & 0.07 \\
\hline 4 & West et al. & $(1994-2001)$ & $1026 / 4,77,627$ & 0.21 \\
\hline 5 & Jensen et al. & $(1997-2007)$ & $40 / 29,276$ & 0.14 \\
\hline 6 & Moshirfar et al. & $(2003-2005)$ & $14 / 20,013$ & 0.07 \\
\hline 7 & Freeman et al. & $(1996-2005)$ & $754 / 4,90,690$ & 0.15 \\
\hline 8 & Ravindran et al. & $(2007-2008)$ & $38 / 42,426$ & 0.09 \\
\hline 9 & Wykoff et al. & $(1995-2009)$ & $8 / 28,568$ & 0.03 \\
\hline
\end{tabular}

Table 1. Reported incidence of acute-onset postoperative endophthalmitis 


\section{Risk factors}

There are concerns that clear corneal sutureless temporal incision cataract surgery may increase the risk of endophthalmitis (Nichamin et al., 2006). In a nosocomial survey (20022009) at a university teaching hospital involving resident, fellow, and faculty surgeons, there was no increase in the incidence of endophthalmitis in the era of sutureless clear corneal cataract surgery (Wykoff et al., 2010). Other more established risk factors for developing acute-postoperative endophthalmitis include patients with diabetes mellitus, cases with posterior capsular rupture and advanced age (Hatch et al., 2009; Doft et al., 2001). A range of risk factors for acute-onset postoperative endophthalmitis is summarized in Table 2.

\section{A. Preoperative:}

1. Diabetes mellitus.

2. Immune compromise.

3. Chronic blepharitis.

4. Infection of the lacrimal drainage system.

5. Contaminated eye drops.

6. Contact lens wear.

7. Contralateral ocular prosthesis.

B. Intraoperative:

1. Application of $2 \%$ Xylocaine gel before povidone-iodine instillation.

2. Prolonged surgery.

3. Secondary IOL.

4. Posterior capsular rupture.

5. Vitreous loss.

6. Contaminated irrigating solution.

7. Clear corneal incision (controversial).

C. Post operative

1. Wound leak.

2. Vitreous incarceration.

Table 2. Risk Factors for Acute-onset Postoperative Endophthalmitis

\section{Classification, isolates and clinical features}

The diagnosis of endophthalmitis is made by the constellation of clinical symptoms and signs. The symptoms and signs vary according to time of presentation (acute-onset postoperative endophthalmitis (APE) (occurring within six weeks of cataract surgery) and chronic postoperative (CPE) endophthalmitis (presenting 6 weeks or more after the surgery) (Johnson et al., 1997).

APE is associated with rapid onset of visual loss, pain, marked intraocular inflammation usually with hypopyon (Figure 2) and caused by rapidly growing bacteria with coagulase-negative Staphylococci (Figure 2) being the most common isolated organism (followed by other Gram positive organisms like Staphylococcus aureus, Streptococcus species and Gram negative bacteria) (Han et al., 1996). Endophthalmitis caused by the 
coagulase-negative staphylococci may have less severe inflammatory signs, often creating difficulty in distinguishing between an infectious and a noninfectious etiology on clinical grounds.

APE, when caused by more virulent organisms such as other Streptococcus species and Gram negative organisms, may be associated with corneal infiltrate, cataract wound abnormalities, afferent pupillary defect, loss of red reflex, initial light perception-only vision, and symptom onset within 2 days of surgery. (Johnson et al., 1997). In CPE caused by less virulent bacteria and fungi, the inflammation is often low-grade and slowly progressive (Table 3). Propionibacterium acnes is the most common organism isolated in CPE (Mezaine et al., 2009; Clark et al., 1999). Other less virulent organisms include Gram positive organisms (coagulase negative Staphyloccocus, Corynebacterium species) (Fox et al., 1991), Gram negative organisms (Alcaligenes xylosoxidans, Acinetobacter calcoaceticus, Ochrobactrum anthropi) (Aaberg et al., 1997; Gopal et al., 2000) and fungi (Candida species, Aspergillus species, and Curvularia species) (Pflugfelder et al., 1988; Pathengay et al., 2006a). Mild to moderate vitritis, white posterior capsular plaque (Figure 3) and keratic precipitates are common clinical signs in CPE (Mezaine et al., 2009; Johnson et al., 1997). Hypopyon is less frequently seen as compared to acute-onset postoperative endophthalmitis (Johnson et al., 1997). The presence of a white plaque inside the capsular bag has been reported in a large series of patients with $P$. acnes endophthalmitis (Nichamin et al., 2006). However, this plaque is not unique to $P$. acnes and has been described in CPE caused by other microorganisms as well (Mezaine et al., 2009; Pathengay et al., 2006a).

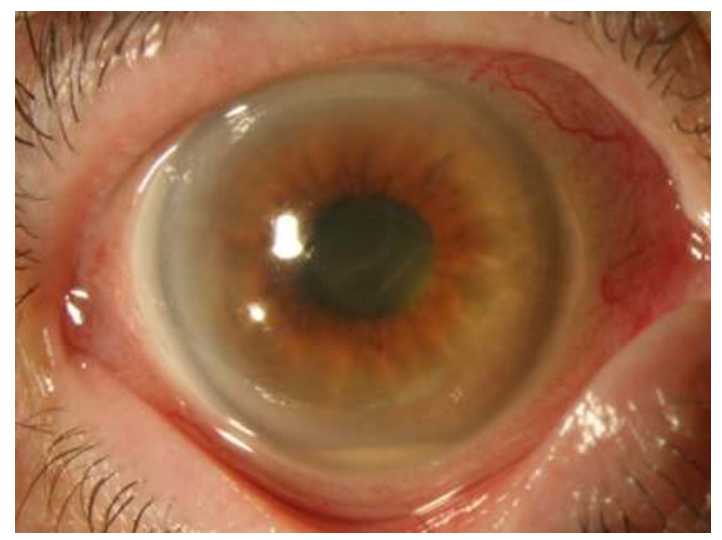

Fig. 1. Acute-onset postoperative endophthalmitis. 


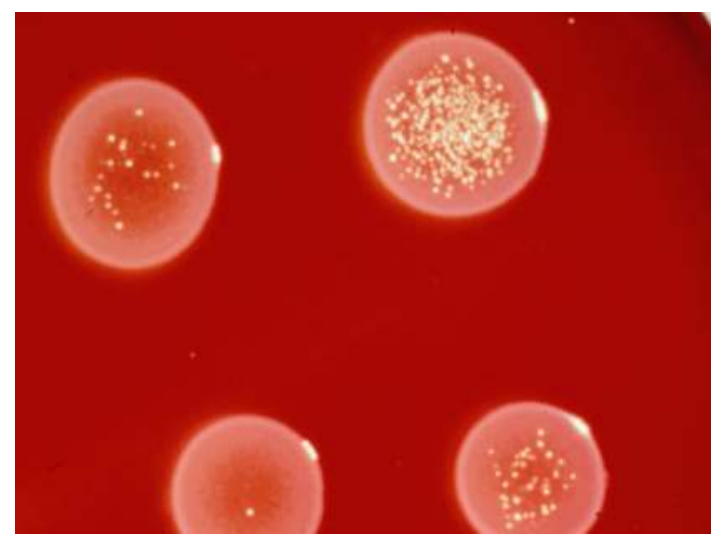

Fig. 2. Coagluase negative Staphylococci isolates from vitreous growing on blood agar.

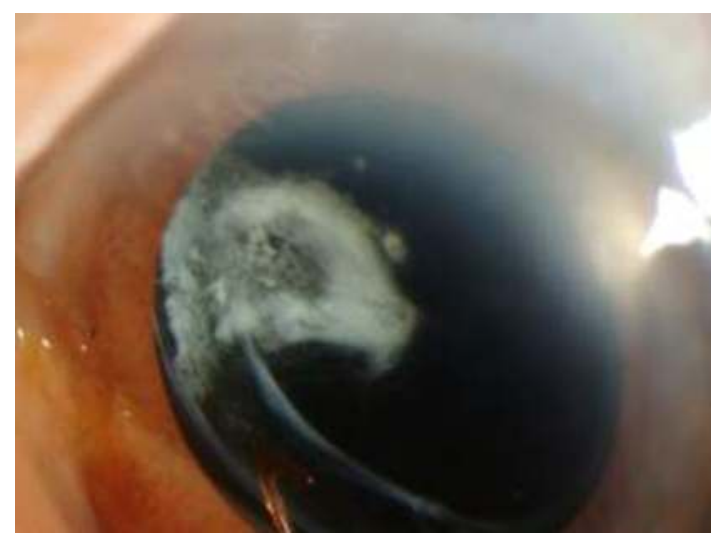

Fig. 3. Delayed-onset postoperative endophthalmitis.

\begin{tabular}{|l|l|}
\hline Bacteria & Fungi \\
\hline P. acnes & Candida parapsilosis \\
\hline S. epidermidis & Other Candida species \\
\hline Corynebacterium species & Paecilomyces species \\
\hline Xanthomonas maltophilia & Aspergillus species \\
\hline Alcaligenes xylosoxidans & Acremonium species \\
\hline
\end{tabular}

Table 3. Bacteria and Fungi commonly associated with delayed postoperative endophthalmitis. 


\section{Differential diagnosis}

The differential diagnosis of endophthalmitis following cataract surgery is summarized in Table 4. Toxic anterior segment syndrome (TASS) can mimic infectious endophthalmitis and is often associated with various toxins entering the eye at the time of surgery (Doshi et al., 2010; Cutler Peck et al., 2010).

The inflammatory response is usually seen with in the first 24 hours after surgery and there is little or no pain. In TASS, corneal edema can be severe and extending "wall to wall". It can be associated with a hypopyon and fibrin formation in the anterior chamber and the vitreous is typically not involved, as is the case with an infectious endophthalmitis. Dehemoglobinized red blood cells in the vitreous cavity can also be confused with endophthalmitis (Nguyen et al., 2006). Retained lens fragments have been reported to cause a marked inflammatory reaction with hypopyon, which may also clinically resemble infectious endophthalmitis (Irvine et al., 1992).
A. Toxic anterior segment syndrome (TASS).
B. Retained lens material.
C. Flare-up of pre-existing uveitis.
D. Dehemoglobinized vitreous hemorrhage.

Table 4. Differential Diagnosis of Endophthalmitis after Cataract Surgery.

\section{Management}

It is important to examine the surgical wound for leakage and vitreous incarceration which may need to be addressed at the time of treatment. If visualization of the posterior segment is limited by either inflammatory material or corneal edema, B-scan echography may help assess the degree of vitreous opacification and determine the presence of either choroidal or retinal detachment.

The treatment of endophthalmitis is usually performed using topical, peribulbar, or retrobulbar anesthesia. A vitreous tap is more commonly utilized but pars plana vitrectomy (PPV) is considered in more advanced cases. The goals of PPV include the following:

1. Obtain intraocular fluid for microbiological analysis (the vitreous specimen generally yields higher culture positivity rates compared to aqueous) (Han et al., 1996);

2. Debulk vitreous of toxins, microorganisms and inflammatory debris.

Although postoperative endophthalmitis is a clinical diagnosis, confirmation by culture of intraocular fluid specimens is an important step in the management. Vitreous can be obtained using a needle (vitreous tap) or by PPV. If a vitreous tap is employed, the use of a 23-gauge butterfly needle (Figure 4) may offer better stability than a straight needle attached directly to a syringe (Raju \& Das, 2004). The undiluted vitreous specimen is then inoculated onto the culture media. The Endophthalmitis Vitrectomy Study reported no significant differences between 3-port PPV versus needle tap/biopsy with respect to microbiologic yield, operative complications, short-term (9-12 months) retinal detachment risk, or visual outcomes (Han et al., 1999). 


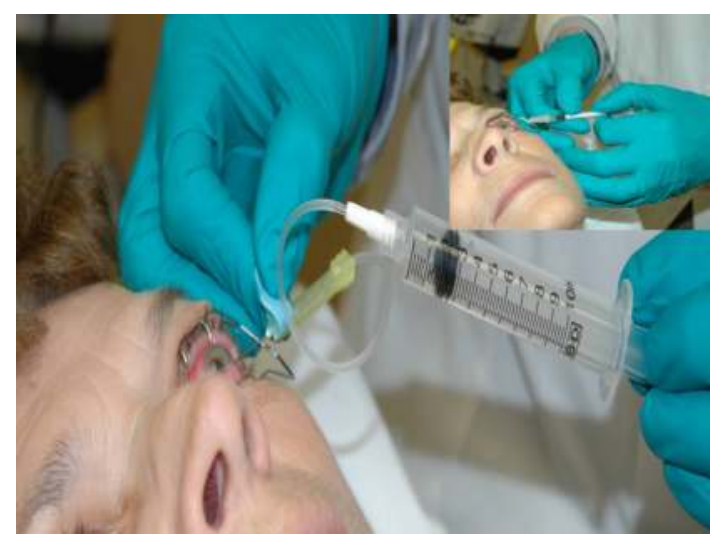

Fig. 4. Butterfly needle used for vitreous tap (upper right: injection of intravitreal antibiotics in separate syringes).

PPV may be performed using small-gauge sutureless (Tan et al., 2008) (Figure 5) or 20gauge vitrectomy techniques and instrumentation. In the Endophthalmitis Vitrectomy Study (EVS), a goal of PPV was the removal of approximately $50 \%$ of the formed vitreous. If the cornea is clear and the pupil dilates well, a more complete PPV can be considered. In typical APE, it is not generally necessary to remove the IOL. In some patients, visibility may be improved by stripping fibrin from the iris or the surface of IOL (Friberg, 1991). In selected cases of delayed postoperative endophthalmitis caused by Propionobacterium acnes or fungi which are not responsive to initial treatment, or when there is recurrence of inflammation, IOL explantation may be considered along with the removal of entire capsular bag (Clark et al., 1999).

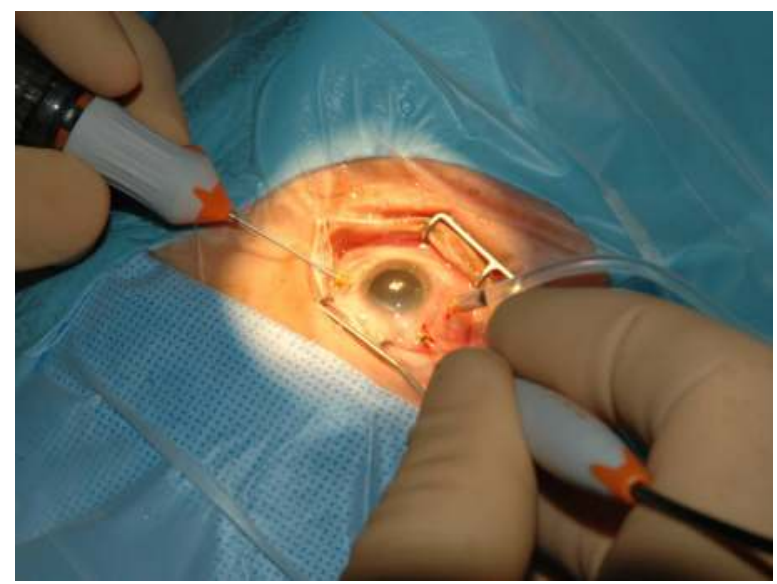

Fig. 5. 23-gauge PPV performed in a patient with acute-onset postoperative endophthalmitis. 
Intravitreal antibiotic therapy (Figure 6) is standard treatment of presumed bacterial postoperative endophthalmitis (Table 5). When endophthalmitis is initially suspected, the pathogen is not known therefore the choice of antimicrobial agent must be made empirically. The EVS reported that the combination of vancomycin $(1 \mathrm{mg} / 0.1 \mathrm{cc})$ and amikacin $(0.4 \mathrm{mg} /$ $0.1 \mathrm{cc})$ were usually effective against the broad spectrum of bacteria causing APE (Endophthalmitis Vitrectomy Study Group, 1995). In the EVS, 100\% of the Gram positive bacteria were susceptible to vancomycin, $89 \%$ of the Gram negative bacteria were susceptible to both amikacin and ceftazidime, and the remaining $11 \%$ were resistant to both amikacin and ceftazidime (Han et al., 1996). However, there are multiple reports of toxicity following administration of intravitreal aminoglycosides, including macular infarction (Campochiaro \& Lim, 1994). An alternative to the aminoglycosides for coverage of Gram negative organisms is the use of intravitreal ceftazidime $2.25 \mathrm{mg}$, a third-generation cephalosporin (Jay et al., 1987; Roth \& Flynn, 1997). Although ceftazidime may precipitate when mixed with vancomycin, this has not been reported to alter its effectiveness in the clinical setting (Kwok et al., 2002). It is generally recommended to use a separate needle and syringe for each antibiotic.

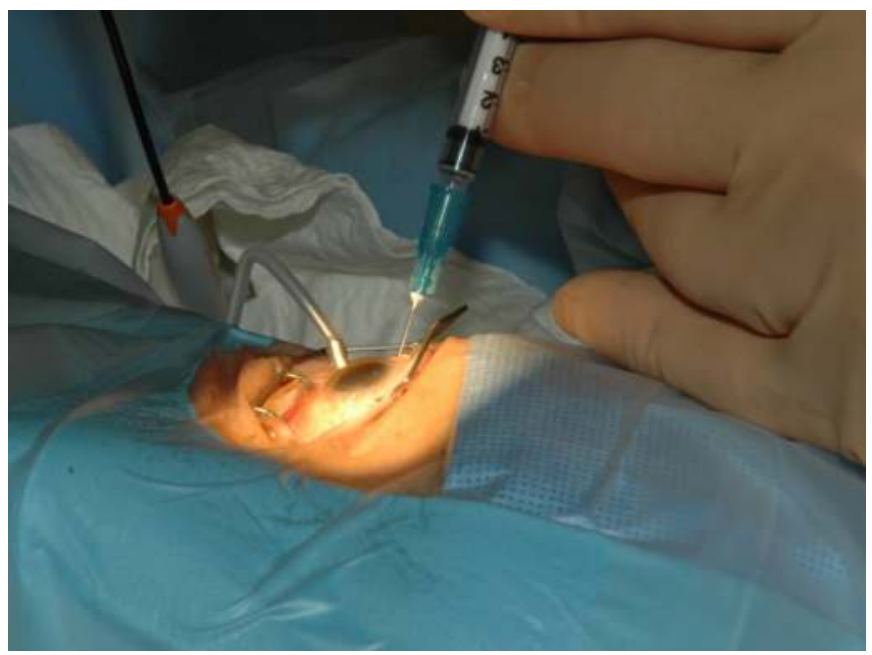

Fig. 6. Intravitreal antibiotics administered at the end of PPV. 

A. Seidel test for potential wound leak
B. Use topical, peribulbar or retrobulbar anesthesia for either vitreous tap or vitrectomy
C. Obtain intraocular specimen
1. 30-gauge needle for anterior chamber tap (optional)
2. 25 or 23-gauge needle for vitreous tap
3. Pars plana vitrectomy (PPV)
D. Preparation of intravitreal antibiotics (usually by pharmacist)
E. Dosages for intravitreal injection
1. Vancomycin $1 \mathrm{mg}$
2. Ceftazidime $2.25 \mathrm{mg}$ or amikacin $0.4 \mathrm{mg}$
3. Dexamethasone $0.4 \mathrm{mg}$ (Optional)

Table 5. Suggested Endophthalmitis Treatment In The Setting Of A Clear Corneal Incision

Intravitreal corticosteroids reduce inflammation-induced ocular damage associated with presumed bacterial endophthalmitis. In a prospective randomized clinical trial of 63 bacterial endophthalmitis cases, intravitreal dexamethasone was reported to reduce inflammation scores early in the course of treatment but had no independent influence on the final visual outcome (Das et al., 1999). In a series of endophthalmitis caused by virulent bacteria such as Gram negative organisms and Staphylococcus aureus, better visual acuity outcomes were reported after adjunctive treatment with intravitreal dexamethasone (Irvine et al., 1992). Intravitreal triamcinolone acetonide has also been reported to reduce inflammation without exacerbating the infection in a small series of endophthalmitis cases (Pathengay et al., 2006b).

Postoperative fungal endophthalmitis more commonly presents as a delayed-onset (chronic) case. Fungi should be suspected in patients that fail to respond to standard intravitreal antibiotic therapy. Traditionally, intravitreal amphotericin B was used commonly to treat suspected fungal endophthalmitis, but intravitreal voriconazole (with or without adjunctive oral therapy) is becoming more frequently employed (Vasconcelos-Santos \& Nehemy, 2009).

\section{Endophthalmitis vitrectomy study}

The EVS was a large multicenter randomized clinical trial comparing vitreous tap to PPV in patients presenting with endophthalmitis within 6 weeks of cataract surgery or secondary IOL implantation (Table 6). A total of 420 patients with APE after cataract surgery was randomly assigned to undergo 3-port PPV or tap/biopsy. They were also randomized to receive either systemic antibiotics (ceftazidime and amikacin) or no systemic antibiotics. At the end of 9 months, patients were assessed for final visual acuity and media clarity.

\subsection{Primary results}

In patients who presented with hand motions (HM) visual acuity or better, there were no statistically significant differences between eyes assigned to vitreous tap versus PPV. In patients who presented with light perception (LP) visual acuity, PPV was associated with significantly more favorable outcomes. The usage of systemic antibiotics was not associated with significant improvements in either visual acuity or media clarity outcomes. (Doft \& Barza, 1996; Barza et al., 1997) 
A. Background - largest series of endophthalmitis after cataract surgery $(\mathrm{N}=420)$

1. EVS enrolled endophthalmitis patients after extracapsular cataract extraction, scleral tunnel phacoemulsification, or secondary IOL within 6 weeks of surgery.

2. Initial results were reported in 1995 and subsequent reports were published in the following years.

B. EVS primary results

1. Patients with LP vision fared better with initial PPV.

2. When patients presented with HM or better vision, equal visual acuity outcomes were reported in the PPV and vitreous tap groups.

3. There was no apparent benefit from the use of systemic antibiotics (amikacin and ceftazidime) compared to controls (no IV antibiotics).

C. EVS secondary results

1. Gram positive bacteria were isolated from $94.2 \%$ patients and Gram negative bacteria in $6.5 \%$. Coagulase-negative micrococci were the most common bacteria isolated in $70 \%$ of patients. Diabetes mellitus was associated with a higher yield of Gram positive, coagulase-negative micrococci.

2. Patients with diabetes mellitus and visual acuity better than LP achieved better visual outcomes with initial PPV (not statistically significant).

3. Additional procedures (as a result of worsening intraocular inflammation or infection or for complications) after the initial treatment: $8 \%$ of PPV eyes and $13 \%$ of tap eyes.

4. There was no statistical difference between PPV and tap groups in terms of microbiological yield, operative complications, retinal detachment $(8.3 \%)$ or visual acuity outcomes (overall, 53\% equal to or better than 20/40 in EVS).

5. In the EVS the incidence of retinal detachment (RD) was $8.3 \%$ and there was no difference in frequency of RD based on whether initial management was PPV or tap.

Table 6. Summary of the Endophthalmitis Vitrectomy Study (EVS)

\subsection{Other results}

Confirmed microbiologic growth was demonstrated in $69.3 \%$ of EVS patients. Undiluted vitreous produced a higher percentage of confirmed positive cultures when compared with aqueous (Barza et al., 1997). Gram positive bacteria were isolated from $94.2 \%$ patients and Gram negative bacteria from $6.5 \%$. Coagulase-negative micrococci were the most common bacteria isolated in $70 \%$ of patients. An additional procedure (as a result of worsening intraocular inflammation or infection or for complications) after the initial treatment was performed in $8 \%$ of PPV eyes and $13 \%$ of tap eyes. (Doft et al., 1998). In the EVS the incidence of retinal detachment (RD) was $8.3 \%$ and there was no difference in frequency of RD based on whether initial management was PPV or tap. (Doft et al., 2000).

Although patients in the EVS derived no demonstrable benefit from these systemic antibiotics, the study made no recommendations regarding treatment with other systemic antibiotics (eg, systemic fluoroquinolones) or systemic antimicrobial agents for other types of endophthalmitis (eg, chronic, bleb-associated, traumatic, fungal, and endogenous forms) (Flynn \& Scott, 2008). Endophthalmitis patients with significant opacification of the anterior chamber precluding the use of PPV, or without light perception visual acuity, were excluded from the EVS. Because these eyes with more severe infection or involving more virulent organisms were 
excluded from the EVS, the effect might have shifted the EVS outcomes to more favorable results. Although the EVS provides general guidelines, the clinician ultimately must decide on the best treatment strategy for the individual patient (Flynn \& Scott, 2008).

The EVS, conducted during 1991-1994, enrolled patients with scleral tunnel incisions. A follow-up retrospective study of patients recruited during 1996-2005 with clear corneal incisions reported generally similar outcomes compared with the EVS (Lalwani et al., 2008).

\section{The European Society of Cataract and Refractive Surgeons (ESCRS) Endophthalmitis Study}

The European Society of Cataract and Refractive Surgeons (ESCRS) Endophthalmitis Study was conducted at 24 centers in 9 European countries from 2003 to 2006 . It was a partially masked, randomized, placebo-controlled study evaluating the prophylactic effect of intracameral cefuroxime injection and/or perioperative levofloxacin eye drops on the incidence of endophthalmitis after cataract surgery. A total of 16,603 patients was recruited in the four arms of the study.

The ESCRS Study reported that the use of intracameral cefuroxime was associated with a significant reduction in the incidence of postoperative endophthalmitis compared to instillation of topical antibiotics. The rate of endophthalmitis was 4.92 times higher in the control group than the cefuroxime-treated group. Perioperative topical levofloxacin was associated with a reduced incidence of endophthalmitis but this difference did not achieve statistical significance. In this study, reported risk factors included clear corneal incisions, silicone IOLs, and surgical complications (Barry et al., 2006; Endophthalmitis Study Group, European Society of Cataract \& Refractive Surgeons, 2007)

\section{Prophylaxis}

The presumed source of the causative organism in the EVS and ESCRS Study was the ocular surface of study patients (Bannerman et al., 1997; Seal et al., 2008). In a systematic review of all prophylactic methods for cataract surgery, instillation of $5 \%$ povidone-iodine on the conjunctiva 3 minutes before the surgery received the highest rating and was the only measure that gained a Grade B rating based on evidence from randomized clinical trials (Ciulla et al., 2002).

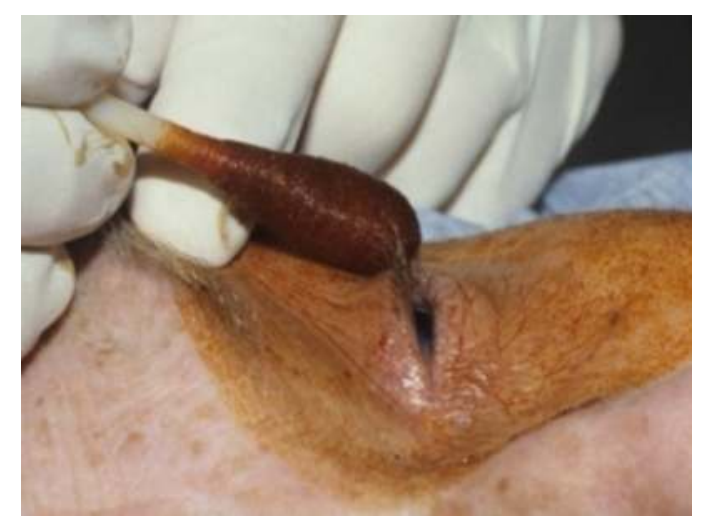

Fig. 7. Povidone-iodine used as prophylaxis preoperatively. 
Povidone-iodine (Figure 7) has reported efficacy in reducing the bacterial load in the conjunctival fornices and is more effective than silver protein solution (Speaker \& Menikoff, 1991). A recent study reported that repeated irrigation of the operative field with povidoneiodine $0.25 \%$ achieved an extremely low bacterial contamination rate in the anterior chamber at the completion of cataract surgery (Shimada et al., 2011).

Preoperative antibiotic administration is associated with a significant decrease in conjunctival bacterial colony counts after the use of certain antibiotics but has not shown superiority to antisepsis with povidone-iodine (Isenberg et al., 1985). A prospective study demonstrated that the addition of moxifloxacin drops had no significant effect in the preoperative reduction of conjunctival bacterial colonization above the effect of povidoneiodine 5\% alone (Halachimi-Eyal et al., 2009).

Antibiotics in the irrigating fluid for cataract surgery have been reported to reduce anterior chamber bacterial contamination rates significantly but their effect on endophthalmitis prevention is less convincing (Sobaci et al., 2003; Srinivasan et al., 2008). The reported lack of effectiveness of both vancomycin and gentamicin in the irrigation fluid may be due to the requirement of more than 140 minutes to exhibit bactericidal effect, whereas the half-life of antibiotics in the anterior chamber is only 51 minutes (Gritz et al., 1996). Intracameral antibiotics have many negative consequences including potential toxicity, increased cost, and the potential for more rapid emergence of resistant bacteria. At least 10 patients in the EVS developed endophthalmitis in spite of receiving antibiotics in the irrigating fluid for cataract surgery.

Other antibiotics which have been used intracamerally include vancomycin, cefazolin and moxifloxacin. Although the safety of moxifloxacin has been reported, there are no published reports about its usage in routine clinical cases (Arbisser, 2008).

Intracameral vancomycin has been reported to significantly reduce the incidence of postoperative endophthalmitis after cataract surgery in a large series from the UK (Anijeet et al., 2010). Since vancomycin is generally reserved for the treating severe lifethreatening or organ-threatening infections, such as endophthalmitis, the United States Centers for Disease Control and Prevention guidelines recommended that this drug should not be used for prophylaxis. Similarly, a study from Spain has reported prophylactic efficacy of intracameral cefazolin, in diminishing the rate of postoperative endophthalmitis without toxic effects on the cornea or retina (Romero et al., 2006). Concerns about the intracameral antibiotic include lack of commercial availability, problems with dosing, $\mathrm{pH}$, and improper constituents within the injection that could lead to postoperative TASS, possible fungal or bacterial contamination during mixing or cystoid macular edema.

Traditionally, subconjunctival antibiotics were commonly administered after intraocular surgery but are less often utilized today. The rationale for subconjunctival antibiotic administration at the completion of the procedure is to inhibit growth of bacteria that may gain entry into the eye. Studies performed evaluating the effectiveness of prophylactic subconjunctival antibiotics in reducing the incidence of postoperative endophthalmitis reported conflicting results (Iyer et al., 2004).

The use of povidone-iodine to reduce the intraoperative bacterial load and the creation of reliably self-sealing incisions to reduce ingress of contaminants are probably the most important of all these factors in reducing the risk of acute postoperative bacterial endophthalmitis. Table 7 summarizes techniques in the reduction of the risk of endophthalmitis after cataract surgery. 


\section{A. Proposed Methods}

1. Preoperative povidone-iodine antisepsis

2. Preoperative topical antibiotics

3. Properly sized and constructed incision

4. Antibiotics in irrigating solution

5. Antibiotics injected into AC

6. Postoperative subconjunctival antibiotics

7. Postoperative topical antibiotics

B. Current Choices for Intracameral Antibiotics

1. Vancomycin

2. Cefuroxime

3. Moxifloxacin

C. Potential Complications of Intracameral Antibiotics

1. Microbial contamination during mixing

2. Incorrect preparation and dosage

3. Allergy

4. Cystoid macular edema

5. Corneal edema

Table 7. Prophylaxis For Endophthalmitis After Cataract Surgery.

\section{Conclusion}

The incidence of endophthalmitis after cataract surgery is variable from about $0.03 \%-0.15 \%$. Povidone-iodine prophylaxis is generally recommended for cataract surgery. Blepharitis, vitreous loss and wound leak are major potential preoperative, intraoperative and postoperative risk factors for endophthalmitis. The significance of clear corneal incisions as a potential risk factor for endophthalmitis remains controversial. The differential diagnosis of endophthalmitis include TASS, retained lens fragments, uveitis and chronic vitreous hemorrhage. The most common organisms causing post-operative endophthalmitis are coagulase negative staphylococci. In the treatment of presumed bacterial endophthalmitis, intravitreal vancomycin and ceftazidime are commonly used for empiric coverage of both Gram positive and Gram negative bacteria. In about $50 \%$ of patients, 20/40 or better visual acuity can be achieved after treatment.

\section{References}

Aaberg, T.M. Jr.; Rubsamen, P.E.; Joondeph, B.C.; \& Flynn, H.W. Jr. Chronic postoperative gram-negative endophthalmitis. Retina. 1997;17:260-2.

Aaberg, T.M. Jr.; Flynn, H.W. Jr.; Schiffman, J.; \& Newton, J. Nosocomial acute-onset postoperative endophthalmitis surgery: a ten-year review of incidence and outcomes. Ophthalmology 1998; 105: 1004-1010.

Allen, H.F.; \& Mangiaracine, A.B. Bacterial endophthalmitis after cataract extraction: a study of 22 infections in 20,000 operations. Arch. Ophthalmol. 1964; 72: 454-462.

Anijeet, D.R.; Palimar, P.; \& Peckar, C.O. Intracameral vancomycin following cataract surgery: An eleven-year study. Clin. Ophthalmol. 2010;4:321-6. 
Arbisser, L.B. Safety of intracameral moxifloxacin for prophylaxis of endophthalmitis after cataract surgery. J. Cataract Refract. Surg. 2008;34:1114-20.

Bannerman, T.L.; Rhoden, D.L.; McAllister, S.K.; Miller, J.M.; \& Wilson, L.A. The source of coagulase-negative staphylococci in the Endophthalmitis Vitrectomy Study: a comparison of eyelid and intraocular isolates using pulsed-field gel electrophoresis: the Endophthalmitis Vitrectomy Study Group. Arch. Ophthalmol. 1997;115:357-361.

Barry, P.; Seal, D.V.; Gettinby, G.; Lees, F.; Peterson, M.; \& Revie, C.W. ESCRS study of prophylaxis of postoperative endophthalmitis after cataract surgery: preliminary report of principal results from a European multicenter study: the ESCRS Endophthalmitis Study Group. J. Cataract Refract. Surg. 2006; 32:407-410.

Barza, M.; Pavan, P.R.; Doft, B.H.; Wisniewski, S.R.; Wilson, L.A.; Han, D.P.; \& Kelsey, S.F. Evaluation of microbiological diagnostic techniques in postoperative endophthalmitis in the Endophthalmitis Vitrectomy Study. Arch. Ophthalmol. 1997;115:1142-50.

Campochiaro, P.A. \& Lim, J.I. Aminoglycoside toxicity in the treatment of endophthalmitis. The Aminoglycoside Toxicity Study Group. Arch. Ophthalmol. 1994; 11:248-53.

Ciulla, T.A.; Starr, M.B.; Masket, S. Bacterial endophthalmitis prophylaxis for cataract surgery: an evidence-based update. Ophthalmology 2002;109:13-24.

Clark, W.L.; Kaiser, P.K.; Flynn, H.W. Jr.; Belfort, A.; Miller, D.; \& Meisler, D.M. Treatment strategies and visual acuity outcomes in chronic postoperative Propionibacterium acnes endophthalmitis. Ophthalmology 1999;106;1665-1670.

Cutler Peck, C.M.; Brubaker, J.; Clouser, S.; Danford, C.; Edelhauser, H.E.; \& Mamalis, N. Toxic anterior segment syndrome: common causes. J Cataract Refract Surg. 2010;36:1073-80.

Das, T.; Jaleli, S.; Gothwal, V.K.; Sharma, S.; \& Naduvilath, T.J. Intravitreal dexamethasone in exogenous bacterial endophthalmitis: results of a prospective randomised study. Br. J. Ophthalmol. 1999;83:1050-5.

Doft, B.H. \& Barza, M. Optimal management of postoperative endophthalmitis and results of the Endophthalmitis Vitrectomy Study. Curr. Opin. Ophthalmol. 1996;7:84-94.

Doft, B.H.; Kelsey, S.F.; \& Wisniewski, S.R. Additional procedures after the initial vitrectomy or tap-biopsy in the Endophthalmitis Vitrectomy Study. Ophthalmology 1998;105:707-16.

Doft, B.M.; Kelsey, S.F.; \& Wisniewski, S.R. Retinal detachment in the Endophthalmitis Vitrectomy Study. Arch. Ophthalmol. 2000 Dec;118:1661-5.

Doft, B.H.; Wisniewski, S.R.; Kelsey, S.F.; \& Groer-Fitzgerald, S. Endophthalmitis Vitrectomy Study Group. Diabetes and postoperative endophthalmitis in the Endophthalmitis Vitrectomy Study. Arch. Ophthalmol. 2001;119:650-656.

Doshi, R.R.; Arevalo, J.F.; Flynn, H.W. Jr.; \& Cunningham, E.T. Jr. Evaluating exaggerated, prolonged, or delayed postoperative intraocular inflammation. Am. J. Ophthalmol. $2010 ; 150: 295-304$.

Endophthalmitis Study Group, European Society of Cataract \& Refractive Surgeons. Prophylaxis of postoperative endophthalmitis following cataract surgery: results of the ESCRS multicenter study and identification of risk factors. J. Cataract Refract. Surg. 2007;33:978-88.

Endophthalmitis Vitrectomy Study Group. Results of the Endophthalmitis Vitrectomy Study. A randomized trial of immediate vitrectomy and of intravenous antibiotics 
for the treatment of postoperative bacterial endophthalmitis. Arch. Ophthalmol. 1995;113:1479-96.

Flynn, H.W. Jr. \& Scott, I.U. Legacy of the endophthalmitis vitrectomy study. Arch Ophthalmol. 2008;126:559-61.

Fox, G.M.; Joondeph, B.C.; Flynn, H.W. Jr.; Pflugfelder, S.C.; \& Roussel, T.J. Delayed-onset pseudophakic endophthalmitis. Am. J. Ophthalmol. 1991;111:163-73.

Friberg, T.R. En bloc removal of inflammatory fibrocellular membranes from the iris surface in endophthalmitis. Arch. Ophthalmol. 1991;109:736-737.

Freeman, E.E.; Roy-Gagnon, M.H.; Fortin, E.; Gauthier, D.; Popescu, M.; \& Boisjoly, H. Rate of endophthalmitis after cataract surgery in Quebec, Canada 1996-2005. Arch Ophthalmol. 2010; 128: 230-234.

Gopal, L.; Ramaswamy, A.A.; Madhavan, H.N.; Saswade, M.; \& Battu, R.R. Postoperative endophthalmitis caused by sequestered Acinetobacter calcoaceticus. Am. J. Ophthalmol. 2000;129:388-90.

Gritz, D.C.; Cevallos, A.V.; Smolin, G.; \& Whitcher, J.P. Jr. Antibiotic supplementation of intraocular irrigating solutions. An in vitro model of antibacterial action. Ophthalmology 1996;103:1204-1208.

Halachimi-Eyal, O.; Lang, Y.; Keness, Y.; \& Miron, D. Preoperative topical moxifloxacin 0.5\% and povidone-iodine $5.0 \%$ versus povidone-iodine $5.0 \%$ alone to reduce bacterial colonization in the conjunctival sac. J. Cataract Refract. Surg. 2009; 35:2109-2114.

Han, D.P.; Wisniewski, S.R.; Wilson, L.A.; Barza, M.; Vine, A.K.; Doft, B.H.; \& Kelsey, S.F. Spectrum and susceptibilities of microbiologic isolates in the Endophthalmitis Vitrectomy Study. Am J Ophthalmol. 1996;122:1-17.

Han, D.P.; Wisniewski, S.R.; Kelsey, S.F.; Doft, B.H.; Barza, M.; \& Pavan, P.R. Microbiologic yields and complication rates of vitreous needle aspiration versus mechanized vitreous biopsy in the Endophthalmitis Vitrectomy Study. Retina 1999;19:98-102.

Hatch, W.V.; Cernat, G.; Wong, D.; Devenyi, R.; \& Bell, C.M. Risk factors for acute endophthalmitis after cataract surgery: a population-based study. Ophthalmology 2009;116:425-430.

Irvine, W.D.; Flynn, H.W. Jr.; Murray, T.G.; \& Rubsamen, P.E. Retained lens fragments after phacoemulsification manifesting as marked intraocular inflammation with hypopyon. Am. J. Ophthalmol. 1992;15;114:610-4.

Isenberg, S.L.; Apt, L.; Yoshmori, R.; Khwarg, S. Chemical preparation of the eye in ophthalmic surgery: IV: comparison of povidone-iodine on the conjunctiva with a prophylactic antibiotic. Arch. Ophthalmol. 1985; 103:1340-42.

Iyer, M.N.; Han, D.P.; Yun, H.J.; Eastwood, D.; Kim, J.E.; Connor, T.B.; Wirostko, W.J.; \& Dev, S. Subconjunctival antibiotics for acute postcataract extraction endophthalmitis: is it necessary? Am. J. Ophthalmol. 2004;137:1120-1121.

Jay, W.M.; Fishman, P.; Aziz, M.; \& Shickley, R.K. Intravitreal ceftazidime in a rabbit model: dose and time dependent toxicity and pharmacokinetic analysis. J. Ocul. Pharmacol. 1987;3:257.

Jensen, M.K.; Fiscella, R.G.; Moshirfar, M.; \& Mooney, B. Third- and fourth-generation fluoroquinolones: retrospective comparison of endophthalmitis after cataract surgery performed over 10 years. J. Cataract Refract. Surg. 2008;34:1460-7. 
Johnson, M.W.; Doft, B.H.; Kelsey, S.F.; Barza, M.; Wilson, L.A.; Barr, C.C.; \& Wisniewski, S.R. The Endophthalmitis Vitrectomy Study. Relationship between clinical presentation and microbiologic spectrum. Ophthalmology. 1997 Feb;104(2):261-72.

Kwok, A.K.; Hui, M.; Pang, C.P.; Chan, R.C.; Cheung, S.W.; Yip, C.M.; Lam, D.S.; \& Cheng, A.F. An in vitro study of ceftazidime and vancomycin concentrations in various fluid media: implications for use in treating endophthalmitis. Invest. Ophthalmol. Vis. Sci. 2002;43:1182-1188.

Lalwani, G.A.; Flynn, H.W. Jr.; Scott, I.U.; Quinn, C.M.; Berrocal, A.M.; Davis, J.L.; Murray, T.G.; Smiddy, W.E.; \& Miller, D. Acute-onset endophthalmitis after clear corneal cataract surgery (1996-2005): Clinical features, causative organisms, and visual acuity outcomes. Ophthalmology. 2008 ;115(3):473-6.

Leopold, I. Incidence of endophthalmitis after cataract surgery. Trans. Ophthalmol. Soc. U.K. 1971; 191: 575-609.

Mezaine, H.S.; Al-Assiri, A.; \& Al-Rajhi, A.A. Incidence, clinical features, causative organisms, and visual outcomes of delayed-onset pseudophakic endophthalmitis. Eur J Ophthalmol. 2009;19: 804-811.

Moshirfar, M.; Feiz, V.; Vitale, A.T.; Wegelin, J.A.; Basavanthappa, S.; \& Wolsey, D.H. Endophthalmitis after uncomplicated cataract surgery with the use of fourthgeneration fluoroquinolones: a retrospective observational case series. Ophthalmology 2007; 114: 686-691.

Nichamin, L.D.; Chang, D.F.; Johnson, S.H.; Mamalis, N.; Masket, S.; Packard, R.B.; \& Rosenthal, K.J. American Society of Cataract and Refractive Surgery Cataract Clinical Committee. ASCRS White Paper: What is the association between clear corneal cataract incisions and postoperative endophthalmitis? J. Cataract Refract. Surg. 2006 ; 32:1556-9.

Nguyen, J.K.; Fung, A.E.; Flynn, H.W. Jr.; \& Scott, I.U. Hypopyon and pseudoendophthalmitis associated with chronic vitreous hemorrhage. Ophthalmic Surg. Lasers Imaging. 2006;37:317-9.

Pathengay, A.; Shah, G.Y.; Das, T.; \& Sharma, S. Curvularia lunata endophthalmitis presenting with a posterior capsular plaque. Indian J. Ophthalmol. 2006a;54:65-6.

Pathengay, A.; Shah, G.Y.; Das, T.; \& Sharma, S. Intravitreal triamcinolone acetonide in the management of exogenous bacterial endophthalmitis. Am. J. Ophthalmol. 2006;141:938-940.

Pflugfelder, S.C.; Flynn, H.W. Jr.; Zwickey, T.A.; Forster, R.K.; Tsiligianni, A.; Culbertson, W.W.; \& Mandelbaum, S. Exogenous fungal endophthalmitis. Ophthalmology. 1988;95:19-30.

Raju, B. \& Das, T. Hyderabad Endophthalmitis Research Group. Simple and stable technique of vitreous tap. Retina.2004; 24: 803-805.

Ravindran, R.D.; Venkatesh, R.; Chang, D.F.; Sengupta, S.; Gyatsho, J.; \& Talwar, B. Incidence of post-cataract endophthalmitis at Aravind Eye Hospital: outcomes of more than 42,000 consecutive cases using standardized sterilization and prophylaxis protocols. J. Cataract Refract. Surg. 2009: 35:4; 629-636.

Roth, D.B. \& Flynn, H.W. Jr. Antibiotic selection in the treatment of endophthalmitis: the significance of drug combinations and synergy. Surv. Ophthalmol. 1997;41:395-401. 
Romero, P.; Méndez, I.; Salvat, M.; Fernández, J.; \& Almena, M. Intracameral cefazolin as prophylaxis against endophthalmitis in cataract surgery. J. Cataract Refract. Surg. 2006;32:438-41.

Seal, D.; Reischl, U.; Behr, A.; Ferrer, C.; Alió, J.; Koerner, R.J.; \& Barry, P. ESCRS Endophthalmitis Study Group. Laboratory diagnosis of endophthalmitis: comparison of microbiology and molecular methods in the European Society of Cataract \& Refractive Surgeons multicenter study and susceptibility testing. J. Cataract Refract. Surg. 2008;34:1439-50.

Shimada, H.; Arai, S.; Nakashizuka, H.; Hattori, T.; \& Yuzawa, M. Reduction of anterior chamber contamination rate after cataract surgery by intraoperative surface irrigation with 0.25\% povidone-iodine. Am. J. Ophthalmol. 2011;151:11-17.

Sobaci, G.; Tuncer, K.; Tas, A.; Ozyurt, M.; Bayer, A.; \& Kutlu, U. The effect of intraoperative antibiotics in irrigating solutions on aqueous humor contamination and endophthalmitis after phacoemulsification surgery. Eur. J. Ophthalmol. 2003;13:773778.

Speaker, M.G. \& Menikoff, J.A. Prophylaxis of endophthalmitis with topical povidoneiodine. Ophthalmology 1991; 98:1769-1775.

Srinivasan, R.; Gupta, A.; Kaliaperumal, S.; Babu, R.K.; Thimmarayan, S.K.; \& Belgode, H.N. Efficacy of intraoperative vancomycin in irrigating solutions on aqueous contamination during phacoemulsification. Indian J. Ophthalmol. 2008;56:399-402.

Tan, C.S.; Wong, H.K.; Yang, F.P.; \& Lee, J.J. Outcome of 23-gauge sutureless transconjunctival vitrectomy for endophthalmitis. Eye 2008; 22:150-151.

Vasconcelos-Santos, D.V. \& Nehemy, M.B. Use of voriconazole in the surgical management of chronic postoperative fungal endophthalmitis. Ophthalmic Surg. Lasers Imaging 2009:40,425-431.

West, E.S.; Behrens, A.;, McDonnell, P.J.; Tielsch, J.M.; \& Schein, O.D. The incidence of endophthalmitis after cataract surgery among U.S. Medicare population increased between 1994 and 2001. Ophthalmology 2005; 112: 1388-1394.

Wykoff, C.C.; Parrott, M.B.; Flynn, H.W. Jr.; Shi, W.; Miller, D.; \& Alfonso, E.C. Nosocomial acute-onset postoperative endophthalmitis at a university teaching hospital (20022009). Am. J. Ophthalmol. 2010 Sep;150(3):392-398. 